\title{
„In der Jungfernheide hinterm Pulvermagazin frequens"
}

\section{Das Handexemplar des Florae Berolinensis Prodromus (1787) von Karl Ludwig Willdenow}

\author{
Katrin Böhme und Staffan Müller-Wille
}

\footnotetext{
„In der Jungfernheide hinterm Pulvermagazin frequens". Karl Ludwig Willdenow's Annotated Copy of Florae Berolinensis Prodromus (1787)
}

\begin{abstract}
We provide a detailed description of an interleaved and heavily annotated copy of Florae Berolinensis Prodromus, a flora of Berlin published by the German apothecary and botanist Karl Ludwig Willdenow in 1787, which today is preserved at the Staatsbibliothek zu Berlin - Preußischer Kulturbesitz. We demonstrate that this is the copy that the author himself used and carried with him during his botanical excursions in and around Berlin to prepare a second edition of the work. By analyzing this document as paper technology, we reveal that even seemingly trifling aspects of its material organization enabled far-reaching biological research agendas that were not originally intended. The hybrid form of manuscript and printed book, used in field excursions, enabled a kind of natural-historical observation that was at once detailed and bound by strict (Linnaean) convention, a combination that inadvertently opened new research questions and suggested new objects of research. We thereby contribute to an understanding of the history of natural history that goes beyond the history of ideas and the intended uses of techniques, giving an example of how routine work on paper within a scientific tradition could generate innovation.
\end{abstract}

Keywords: Karl Ludwig Willdenow, Carl von Linné, eighteenth-century natural history, history of taxonomy, history of pharmacology

Schlüsse/wörter: Karl Ludwig Willdenow, Carl von Linné, Naturgeschichte des 18. Jahrhunderts, Geschichte der Taxonomie, Geschichte der Pharmazie

Das Zitat im Titel entstammt den handschriftlichen Anmerkungen, mit denen der Berliner Botaniker Karl Ludwig Willdenow (1765-1812) das Handexemplar seines 1787 erschienenen "Vorboten einer Flora Berlins“ (Florae Berolinensis Prodromus) versah. Die Notizen dokumentieren Willdenows botanische Streifzüge durch das Berliner Stadtgebiet und Umland. Meist wird hier schlicht festgehalten, wo genau er eine bestimmte Pflanzenart angetroffen hat und wie häufig sie dort vertreten war - in dem hier betrachteten Fall, die Schwarzschopf-Segge aus der Familie der Sauergrasgewächse oder Riedgräser 
(Cyperaceae), die Willdenow erstmals beschrieb und mit dem botanischen Namen Carex paradoxa belegte. ${ }^{1}$

Die wortkarge Genauigkeit, die aus seinen Aufzeichnungen spricht, passt zu Willdenows beruflichem Hintergrund als Apotheker (Schlechtendal 1814). Inventarlisten über die vorrätig gehaltenen, meist pflanzlichen Heilmittel, Bestellungen und Rezepturen sowie doppelte Buchführung gehörten seit der Renaissance zur Arbeitswelt der Apotheker. Elemente dieser Verzeichnispraktiken fanden Eingang in die gelehrte Welt der Naturgeschichte, so wie umgekehrt Apotheker aus naheliegenden Gründen von früh an zu den wichtigsten Konsumenten naturhistorischer Literatur gehörten (Pugliano 2012). Lokalfloren wie die Berliner Flora Willdenows bildeten in desem Zusammenhang ein Genre. Sie entstanden ab dem frühen 17. Jahrhundert meist in Universitätsstädten und dienten Medizinstudenten als papierne Grundlage zur Ein- und späteren Ausübung von Mikropraktiken des Notiznehmens und der Bezugnahme, Entdeckung und Ergänzung von Neuem sowie der Korrektur bestehenden Wissens (Cooper 2007: 72-79).

In diesem Beitrag wollen wir Willdenows annotiertes Handexemplar in seiner Funktion als Forschungswerkzeug vorstellen. ${ }^{2}$ Der Band befindet sich heute im Besitz der Staatsbibliothek zu Berlin - Preußischer Kulturbesitz. ${ }^{3}$ Dass es sich dabei um Willdenows eigenes Exemplar handelt, erschließt sich aus einem Vermerk in den sogenannten Inventarien, die vom Oberbibliothekar Friedrich Wilken (1777-1840) seit 1819 erstellt wurden. Mittels eines Handschriftenvergleichs konnten wir dies bestätigen. ${ }^{4}$ Der Band ging vermutlich 1818 in den Besitz der damaligen Königlichen Bibliothek über, als es Willdenows Witwe mit Unterstützung des Berliner Botanikers Heinrich Friedrich Link (1767-1851) gelang, die Bibliothek und das Herbarium ihres Mannes an den preußischen Staat zu verkaufen. ${ }^{5}$ Das etwa 20.260 Arten umfassende Willdenow-Herbar bildete dann den Grundstock des 1815 mit Links Amtsantritt gegründeten Königlichen Herbariums (Hiepko 1972). Während das Herbar noch heute im Botanischen Museum als geschlossene Sammlung genutzt werden kann, wurde seine Bibliothek in den Gesamtbestand der Königlichen Bibliothek integriert.

Willdenows Handexemplar ist erstaunlich gut erhalten, bis auf den Einband, der Mitte der 1960er Jahre durch einen spartanischen Halbgewebeeinband mit sogenannter Elefantenhaut (ein mattgraues, geleimtes Papier) ersetzt wurde. Der Buchblock des Oktavbandes setzt sich aus den gedruckten Seiten des Buches sowie aus eingebundenen und mit handschriftlichen Notizen versehenen Blättern zusammen. Das Material lässt sich drei zeitlichen Horizonten zuordnen:

Den ersten Horizont bildet ein fünf Blätter umfassendes, zwischen Vorwort und Hauptteil des gedruckten Textes befestigtes Manuskript Willdenows (Abb. 1). ${ }^{6}$ Es ist vermutlich vor Drucklegung der ersten Auflage des Prodromus entstanden, und enthält einen Auszug aus der 1663 erschienenen Flora 
Marchica von Johann Sigismund Elsholtz (1623-1688), dem „Hofmedicus“ von Kurfürst Friedrich Wilhelm. Willdenow listet hier zunächst „Arzneykräuter, welche von selbst hier zu Lande wachsen“ auf (bei Elsholtz durch ein Sternchen gekennzeichnet) und schließt dann eine Liste an, in der er die „[w]ilde[n] Kräuter, welche auf den Apotheken insgemein nicht gebraucht werden " aufzählt. ${ }^{7}$ Die Einträge beider Listen sind in Zehnerschritten gezählt und führen 158 beziehungsweise 360 Arten auf. Letztere Zahl erscheint auch in einer kurzen, handschriftlichen Rechnung am Rand der letzten Seite des Vorworts des Prodromus. ${ }^{8}$

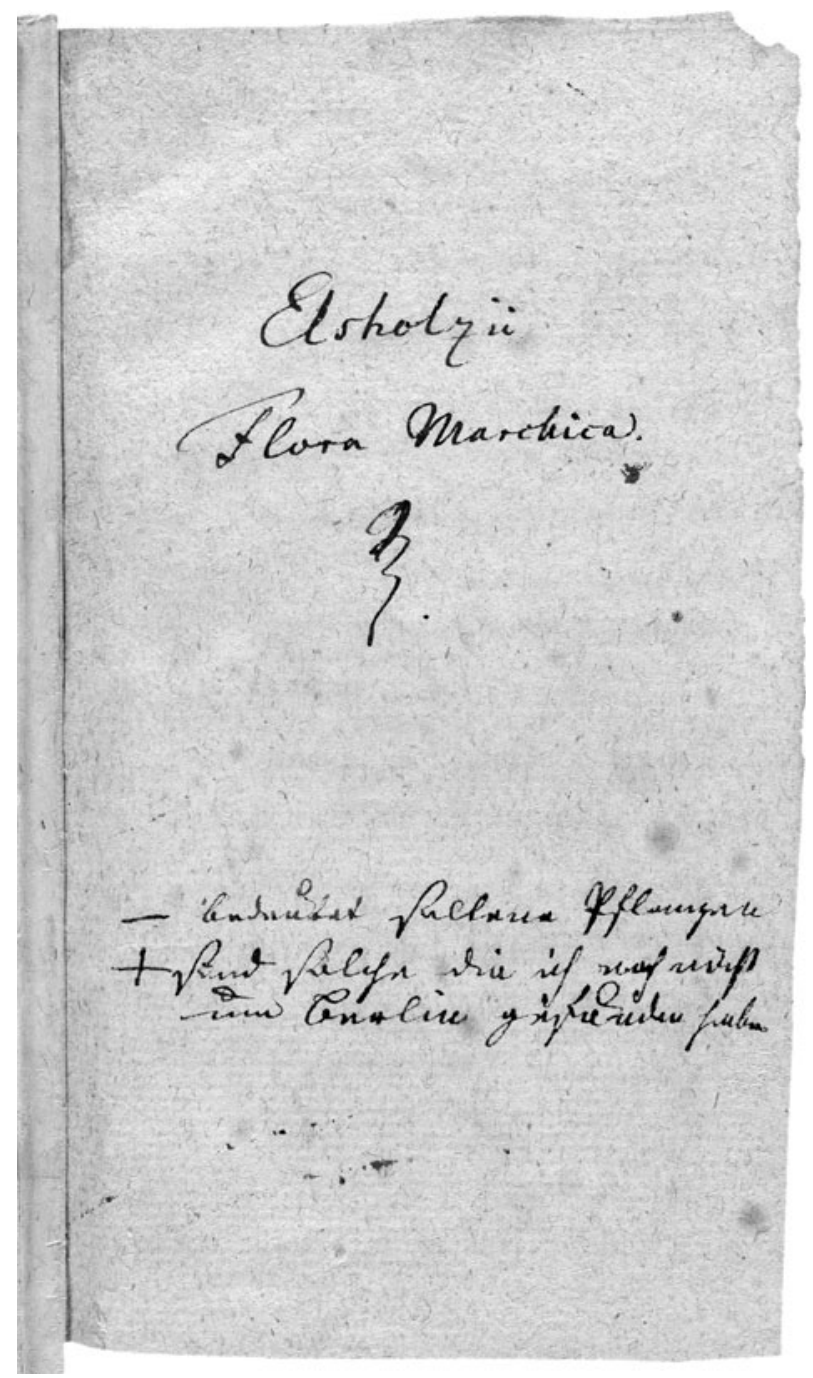

Abb. 1 Titelseite eines handschriftlichen Auszugs der Flora Marchica von Elsholtz 1663 in Willdenow 1787 (SBB, Abteilung Historische Drucke, mit freundlicher Genehmigung der Staatsbibliothek zu Berlin - Preußischer Kulturbesitz). 
Manchen Artnamen in den beiden Listen folgt ein Minuszeichen, was laut einer Notiz auf dem Titelblatt des Manuskripts bedeutet, dass es sich um eine "besonders seltene" Art handelt. Anderen folgt ein Pluszeichen, das angibt, dass es sich um eine Art handelt, „die ich [Willdenow] noch nicht um Berlin gefunden habe “. 9 Ein Vergleich mit Willdenows Quelle ergibt außerdem, dass er die von Elsholtz verwendeten Pflanzennamen durch Linné'sche binäre Artnamen ersetzte und dabei die Zahl der Arten reduzierte, indem er von Elsholtz erwähnte Varietäten zu einer Art zusammenführte. Auf der Rückseite des letzten Blattes führt Willdenow unter der Überschrift „Arenstorff“ noch weitere 19 Pflanzenarten auf, für die jeweils Fundorte aus der Umgebung Berlins angegeben sind („Berberis vulgaris - bey Weißensee “). ${ }^{10}$ Diese kurze Artenliste bezieht sich auf die 1762 erschienene Pharmacopöe eines C. F. Arenstorff, der ab 1738 als Geselle in der Berliner Hofapotheke tätig war (Arenstorff 1762: Vorrede, unpaginiert). Wie Willdenow im Vorwort zum Prodromus festhält, sind dies die beiden einzigen früheren Publikationen, die der Flora Berlins gezielt gewidmet sind. Bei dem Manuskript handelt es sich also um eine sorgfältige und systematische Auswertung älterer Literatur zur Flora Berlins, die den Stand der Forschung zusammenfasst und vermutlich zur Vorbereitung des Prodromus diente. Aus ebendiesem Grund hob Willdenow die Liste wohl auch in seinem Handexemplar auf, denn sie dokumentiert, wie weit er mit seinem Prodromus über das bisher Erreichte hinausgegangen war.

Der zweite Zeithorizont besteht aus den Blättern des gedruckten Buches, das zur Ostermesse 1787 erschien, also noch vor dem 22. Geburtstag des Autors (Allgemeines Verzeichnis 1787: 627). Der vollständige, lateinische Titel lautet in deutscher Übersetzung: „Vorbote zu einer Flora Berlins zusammengestellt nach dem vom berühmten Mann und Ritter C. P. Thunberg verbesserten Linné’schen System." Der Buchtext gliedert sich in ein elfseitiges Vorwort, den systematischen, 422 Seiten umfassenden Hauptteil, einen Index der Gattungsnamen und ihrer Synonyme, einen Tafelteil und schließlich ein Verzeichnis von hinzuzufügenden Arten (Addenda) und Druckfehlern (Sphalmata typographica). Wie der Titel des Buches verrät, verzeichnet Willdenow die in Berlin vorkommenden Arten nach Linnés Sexualsystem in der veränderten Fassung von dessen Schüler Carl Peter Thunberg (17431828). Der Bezug auf dieses System erlaubt Willdenow, weitgehend auf langwierige Beschreibungen und Synonymielisten zu verzichten. Die meisten Einträge im Prodromus zitieren bloß den Linnéschen Trivialnamen und die Diagnose der jeweiligen Art aus Linnés Systema naturae, ergänzt um Bandund Seitenangabe und gegebenenfalls zu unterscheidende Varietäten, so dass sich Leser wegen weiterer Details an dieses weit verbreitete Werk wenden konnten. Dem hat Willdenow dann in der Regel nur noch hinzugefügt, wo genau in Berlin diese Art anzutreffen ist, in welchem Monat sie blüht, und unter welchem Namen sie in Apotheken gehandelt wird. ${ }^{11}$ Nur in einigen Fällen wich er von diesem Schema $a b$, indem er nomenklatorische und 
klassifikatorische Entscheidungen kurz begründet, morphologische Besonderheiten hervorhebt, oder ausführlichere, oft durch eine Tafelabbildung ergänzte Beschreibungen zuvor nicht beschriebener oder neu entdeckter Arten aufnahm. ${ }^{12}$ Die Arten sind durchgängig nummeriert, und im Durchschnitt nimmt jeder der 1.243 Arteinträge jeweils rund eine Viertelseite ein. So wird die Berliner Pflanzenvielfalt in einem typographisch klar und übersichtlich gegliederten Raum präsentiert, auf dessen diskrete Einheiten mit Hilfe des verwendeten Klassifikationssystems und des angefügten Gattungsindex rasch und zuverlässig zugegriffen werden kann.

Den dritten Zeithorizont bilden schließlich handschriftliche Korrekturen und Anmerkungen Willdenows im gedruckten Text sowie auf den Blättern, mit denen der systematische Hauptteil und der Nachsatz des Handexemplars durchschossen wurden. Als „Durchschuss“ bezeichnet man leere Blätter, die vom Buchbinder zwischen die Seiten des ungebundenen Buchblocks eingelegt werden, und zwar so, dass einer bedruckten Buchseite immer eine leere Seite gegenübersteht, auf der sich dann bequem handschriftliche Anmerkungen zum nebenstehenden, gedruckten Text machen lassen. Willdenow folgte damit einer Praxis, die seit der Renaissance unter gelehrten Lesern verbreitet war (Blair 2004: 92; Brendecke 2005), und die vor ihm bereits Linné verwendet hatte, um mit großer Effektivität naturhistorische Beobachtungen zu sammeln (Müller-Wille/Charmantier 2012). Demnach lässt sich von den Aufzeichnungen auf den Durchschussblättern annehmen, dass sie nach der Drucklegung entstanden sind. Diese Annahme wird durch den Inhalt der Aufzeichnungen bestätigt. Schon auf der Vorderseite des ersten Durchschussblattes heißt es nach einem Notabene, dass ,in der zweiten Auflage die Gattungen der Umbelliferen und Pilze zu verbessern sind“, und dass „die Einteilung der Gattungen der Algen, z. B. Lichen, Verrucar[ia] usw. aus sichereren Merkmalen zu entnehmen ist."13 Offenbar plante Willdenow also eine zweite, überarbeitete und erweiterte Auflage seiner Berliner Flora, zu der es dann allerdings vor seinem Tod im Jahre 1812, kurz nach seiner Berufung auf den Lehrstuhl für Botanik an der gerade gegründeten Berliner Universität, nicht mehr kam. Dass das durchschossene Exemplar der Vorbereitung einer geplanten Neuauflage dienen sollte, zeigt auch der Umstand, dass sich die meisten Aufzeichnungen als inhaltliche Ergänzungen des gegenüberstehenden, gedruckten Textes verstehen lassen. So fügte Willdenow auf den Durchschussblättern weitere Fundorte oder Literaturangaben hinzu, und zwar immer auf derselben Höhe mit der im Druck aufgeführten Art, der diese Ergänzungen galten. ${ }^{14}$ In einigen Fällen beschrieb er, wie wir am Beispiel der Schwarzschopf-Segge zeigen werden, darüber hinaus neue Arten, die er auf den Durchschussblättern gegenüber den Arteinträgen derselben Gattung notierte. Ein handschriftlicher Index auf den Durchschussblättern des Nachsatzes erlaubt den Zugriff auf diese Beschreibungen. Tinte und Schriftbild all dieser Annotationen variieren stark, so dass man den Eindruck gewinnt, 
dass sie über einen längeren Zeitraum und unter verschiedenen Umständen erfolgt sind. Zuweilen finden sich auch Moosreste, und zwar an Stellen, an denen das Papier verfärbt und stärker feucht geworden ist. Das ist ein Hinweis darauf, dass das Handexemplar auch genutzt wurde, um für kurze Zeit, gesammelte Pflanzenbelege aufzunehmen. ${ }^{15}$ Schließlich gibt es noch einen kleinen Zettel, auf dem einige Pflanzennamen verzeichnet sind. Der verschmutzte Rand lässt vermuten, dass dieser Zettel als Lesezeichen benutzt wurde. ${ }^{16}$ All dies verrät, dass Willdenow den Band auf seinen botanischen Exkursionen in und um Berlin mit sich führte und für die Aufzeichnung neuer Beobachtungen nutzte. Stadtgebiet und Umland wurden gewissermaßen botanisch kartographiert, was dann zu unzähligen Fundortnotizen wie „auf dem Juden Kirchhoff", „ad Wilmersdorf / Rüdersdorf / Thiergarten rarius“, „in den Papenbergen passim / Tegel / Thiergarten“ oder „im Dustern Keller frequens" führte. ${ }^{17}$ Für Willdenow war der Band offenbar kein kostbares Einzelstück, sondern ein Arbeitsmittel für den alltäglichen Gebrauch im Feld.

Die drei im Vorhergehenden beschriebenen Zeithorizonte sind von unterschiedlicher Temporalität. Das Elsholtz-Manuskript entstand im Rückblick und reduziert vergangenes Wissen über die Berliner Flora zu einem systematischen Überblick, der von ursprünglichen Kontexten und Intentionen abstrahiert. Erst so wurde für Willdenow deutlich erkennbar, wie viele neue Arten er in seinem Prodromus dokumentierte. Der gedruckte Text des Werks selbst liefert einen Zwischenstand, eine durch die Drucklegung festgehaltene Momentaufnahme, die sich anschließend als Plattform für die Fortschreibung der Berliner Flora nutzen ließ. Dazu schließlich dienen die Durchschussblätter, deren Notizen ergebnisoffen angelegt sind, aber durch die Gegenüberstellung mit dem gedruckten Text strukturiert werden. Willdenows Handexemplar diente also gleichzeitig als Referenzwerk und Notizbuch sowie vermutlich sogar als kurzfristige Pflanzenpresse auf seinen Exkursionen. So ließen sich gesammelte Informationen gleichsam am richtigen „Ort“ des Pflanzensystems verwahren und zu einem späteren Zeitpunkt gezielt abrufen und auswerten.

Am Beispiel der Schwarzschopf-Segge wollen wir dies kurz ausführen (Abb. 2): Sie gehört zu den neuen, weder von Elsholtz noch Arenstorff beschriebenen Arten, für die Willdenow im Durchschuss seines Handexemplars eine ausführliche, mehr als zwei Drittel des Blattes einnehmende, handschriftliche Beschreibung aufnahm. Vorangestellt ist dieser Beschreibung eine neue, laufende Nummer („n. 1308“), der Artname Carex paradoxa (wobei der Gattungsname doppelt und das Artepitheton einfach unterstrichen sind), eine kurze Artdiagnose („spicis androgynis et sexu distinctis culmo triquetro“) sowie in einem neuen Absatz die im Titel unseres Beitrags zitierte Notiz über das Vorkommen, die zu einem späteren Zeitpunkt um ein weiteres Vorkommen ergänzt wurde (,in der Lipe im Grunewald frequens"). Dieses Format lehnt sich bis in schriftbildliche Einzelheiten wie Hervorhebungen und Platzierung von Absätzen an das Format der Arteinträge im gedruckten Text an. 
In Willdenows Herbarium sind insgesamt sieben Belegexemplare $\mathrm{zu}$ Carex paradoxa erhalten. Willdenow bewahrte diese ursprünglich in Umschlägen auf. Sie wurden nach seinem Tod von Franz Leonhard Schlechtendal (1794-1866) geordnet und auf Herbarblätter montiert, wobei handschriftliche Notizen Willdenows aufbewahrt wurden (Hiepko 1972: vii). Eine dieser Notizen - von Schlechtendal auf dem blauen Umschlag befestigt, der die Belegexemplare enthält - besteht aus dem kryptischen Vermerk „Car[ex] flav[a] 1368 $\beta$ “. Unter diesem Verweis befindet sich ein kleiner, horizontaler Riss im Papier, was darauf hindeutet, dass der Zettel ursprünglich an den Stiel eines Belegexemplars geheftet war. Im Handexemplar des Prodromus findet sich ein Eintrag für die Art Carex flava genau auf der Seite, der Willdenows handschriftliche Beschreibung von Carex paradoxa gegenüber steht. Bei der Zahl „1368“ dürfte es sich um einen irrtümlichen Verweis auf die laufende Nummer von Carex paradoxa im Handexemplar handeln. Insgesamt sprechen diese Indizien dafür, dass Willdenow Belegexemplare im Feld sammelte, mit Etiketten versah und erst später genaue taxonomische Zuordnungen und Beschreibungen in seinem Handexemplar vornahm.

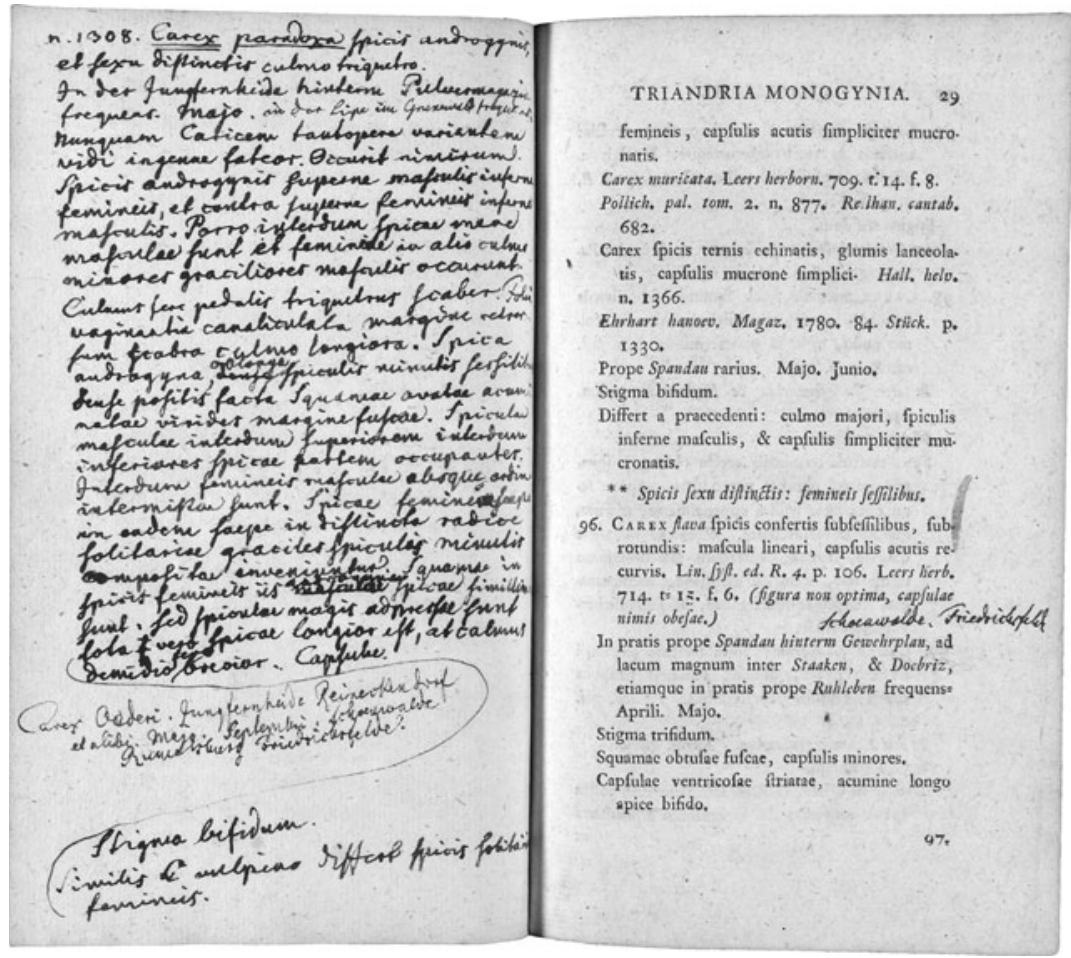

Abb. 2 Beschreibung von Carex paradoxa in Willdenow 1787: opp. 29 (SBB, Abteilung Historische Drucke, mit freundlicher Genehmigung der Staatsbibliothek zu Berlin Preußischer Kulturbesitz). 
Ursprünglich, so scheint es, hatte er Carex paradoxa für eine Varietät der bereits früher beschriebenen Art Carex flava gehalten.

Die laufende Nummer 1308 ergibt sich aus der Fortführung der Artennummerierung des Prodromus. Auf dem Durchschussblatt vor den Addenda sowie im Nachsatz des Bandes findet sich eine handschriftliche „Übersicht über die hier neuerlich beobachteten Pflanzen (Conspectus vegetabilum nuperrime heic observatorium [sic]"), in der die Namen der Arten aufgelistet sind, die im Durchschuss neu beschrieben wurden (Abb. 3). Die Nummerierung schließt unmittelbar an die letzte, im Prodromus vergebene Nummer (1243) an und erreicht mit den neu hinzugekommenen Arten 1378. Das bedeutet, dass zu den 1243 schon im Prodromus von 1787 veröffentlichten Berliner Arten noch einmal 135 hinzugekommen sind. Zu jedem Artnamen gehört außerdem ein Verweis auf die Seitenzahl, an der die neu aufgenommene Art in den Prodromus eingerückt wurde. Die Reihenfolge der Arten in dieser Liste ist nicht systematisch und spiegelt daher nicht die Reihenfolge der Beschreibungen im Band selbst wider. So kommt es, dass Carex paradoxa als Nr. 1308 neben den Arten Nr. 95 (Carex Leersi) und 96 (Carexflava) aufgeführt wird. Es ist deshalb anzunehmen, dass die Liste nach und nach mit der Entdeckung neuer Arten entstand, und auch die neuen Nummern keinem anderen Prinzip folgend vergeben wurden. ${ }^{18}$

Die Artdiagnose, die Willdenow für Carex paradoxa liefert, ist bemerkenswert. Sie begründet auch die Position, die diese Art im Handexemplar Willdenows zwischen Carex Leersi und Carex flava einnimmt. Carex ist eine sehr artenreiche Gattung (der Prodomus zählt allein 31 Arten) und Willdenow gliederte sie daher in zwei Gruppen: Seggen mit zweigeschlechtlichen Ähren („spicis androgynis") und Seggen mit getrenntgeschlechtlichen Ähren (,spicis sexu distinctis"). Die Artdiagnose von Carex paradoxa besagt nun allerdings, dass es sich hierbei um eine Art handelt, die neben einem „dreikantigen Halm (culmo triquetro)" durch Ähren ausgezeichnet ist, die sowohl zweigeschlechtlich als auch getrenntgeschlechtlich sind („spicis androgynis et sexu distinctis“). Carex Leersi ist die letzte der unter den „zweigeschlechtlichen“ Seggen aufgeführten Arten, während Carex flava die erste Art ist, die von Willdenow unter den "getrenntgeschlechtlichen“ Seggen aufgelistet wird. Carex paradoxa - oder die „seltsame Segge“, wie Willdenow sie in der vierten, von ihm selbst besorgten Auflage von Linnés Species plantarum nennt (Linné [1805] 1797-1830, Bd. 4: 243) - nimmt also vor dem Hintergrund der taxonomischen Unterscheidungen, die der Einteilung der Arten im Prodromus zu Grunde liegen, eine ambivalente Position ein, die durch ihre Platzierung, aber auch durch das gewählte Artepitheton paradoxa, genau erfasst wird.

Damit untergräbt Carex paradoxa allerdings ausgerechnet eine der Unterscheidungen, die für das Linné'sche Sexualsystem maßgeblich sind. Dieses System klassifizierte Pflanzen nach der Anzahl und Anordnung männlicher und weiblicher Geschlechtsorgane. Linné war noch davon 


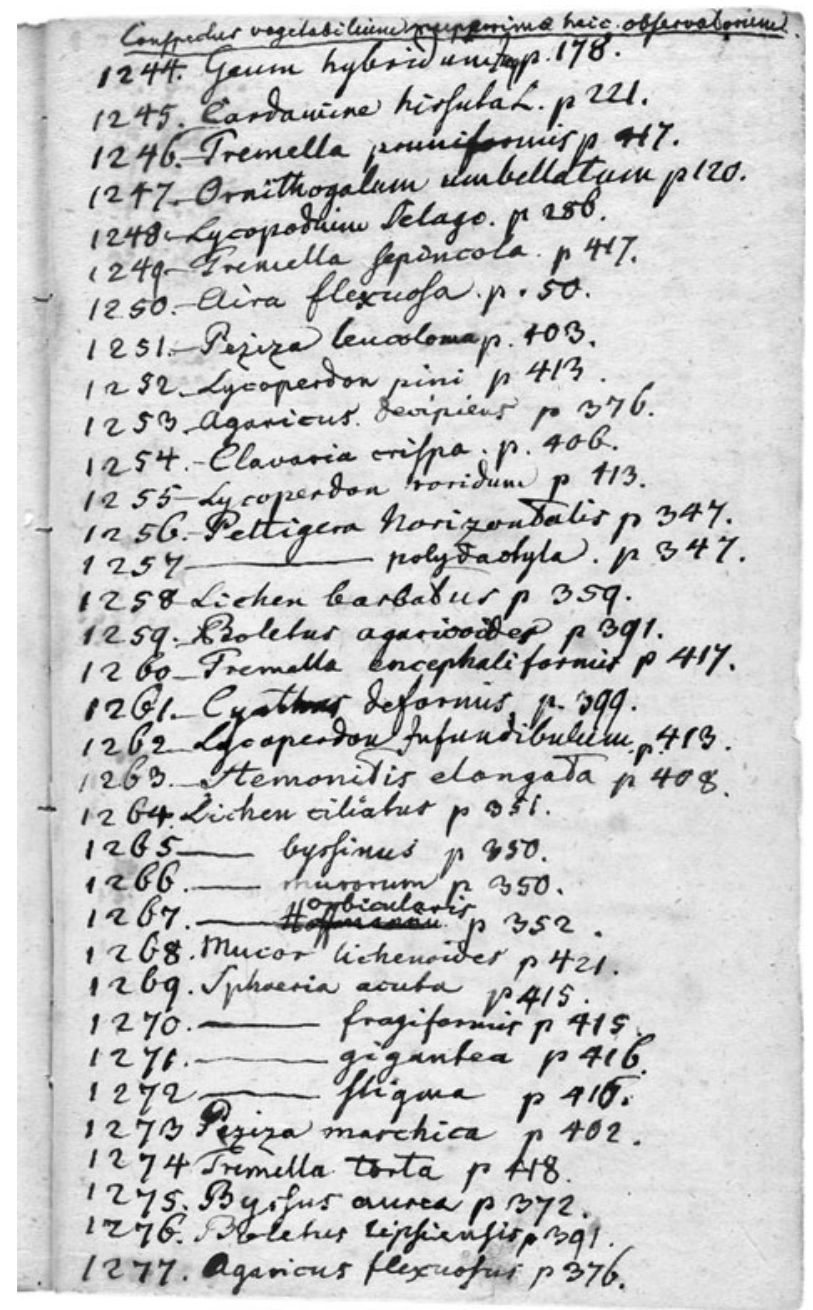

Abb. 3 Conspectus vegetabilum nuperrime heic observatorium in Willdenow 1787, Nachsatz (SBB, Abteilung Historische Drucke, mit freundlicher Genehmigung der Staatsbibliothek zu Berlin - Preußischer Kulturbesitz).

ausgegangen, dass es sich dabei um besonders verlässliche Unterscheidungskriterien handelte. „Ich bekenne freimütig“, heißt es dagegen gleich zu Beginn der handschriftlichen Beschreibung von Carex paradoxa im Handexemplar, „daß ich niemals eine Segge gesehen habe, die so sehr [in ihren Geschlechtsmerkmalen, K.B/S.M-W.] variiert."19 Dies wird auch der Grund sein, warum Willdenow ganze sieben Belegexemplare von Carex paradoxa sammelte. $\mathrm{Zu}$ einer zweiten Auflage des Prodromus kam es, wie bereits erwähnt, nicht mehr, aber 1794 trug Willdenow in der Königlichen Akademie der Wissenschaften eine Abhandlung „Über die in der Gegend von Berlin wildwachsenden Rietgras-Arten“ vor, die dann 1799 in der Zeitschrift der Akademie erschien. 
Dieser Aufsatz enthielt auch eine stark erweiterte Beschreibung von Carex paradoxa. Noch einmal betont Willdenow hier in einem als „Beobachtung (Observatio)“ gekennzeichneten Absatz, dass „das Geschlecht in dieser Art sehr unbeständig" sei. ${ }^{20}$ Dennoch bleibt es auch in diesem Aufsatz bei einer Einteilung der Carex-Arten nach der Verteilung von männlichen und weiblichen Blüten auf Ähren. Sie ist allerdings so angepasst, dass die „seltsame“ Segge ihren eindeutigen Platz findet: Carex paradoxa gehört nun ganz einfach zu den Seggen, „die mehrere mit männlichen und weiblichen Blumen vermischte Aehren haben“" (Willdenow 1799: 37).

Wenn dies wie eine Ad-hoc-Lösung erscheint, so lohnt ein Blick auf die kurze Diskussion, die Willdenow im Vorwort des Prodromus Fragen der Taxonomie widmet. Zwar sei, so heißt es dort, das Sexualsystem mit seinen linear aufeinanderfolgenden Unterscheidungen grundsätzlich nicht in der Lage, den „gleichsam netzförmigen Zusammenhang der einzelnen Geschöpfe“ wiederzugeben (Willdenow 1787: x). Aber das Linné’sche System „trage das Siegel der Menschheit vor sich her", bilde also ein durch Konvention zustande gekommenes, soziales System von Bezugspunkten (ebd.: xi). Fälle wie Carex paradoxa machen deutlich, dass es gerade dieses Bezugssystem war, vor dem sich die netzförmigen, taxonomische Unterscheidungen gegenlaufenden Verwandtschaftsbeziehungen unter Pflanzen sowie die natürliche Variabilität scheinbar wesentlicher Merkmale abheben konnten. Ohne diesen Bezugspunkt wäre diese Varibilität allenfalls ein isoliertes Kuriosum, aber kein Anlass zu generalisierenden Überlegungen. So bezog sich Willdenow unter anderem auf die Gattung Carex, als er in seinem Grundriss der Kräuterkunde behauptete, , dass nichts unbeständiger als das Geschlecht“ sei (Willdenow 1792: 214). Charles Darwin sollte ein knappes Jahrhundert später in seinem Buch The Effects of Cross and Self Fertilisation in the Vegetable Kingdom den evolutionären Wurzeln dieser „Unbeständigkeit“ nachgehen (Darwin 1876). Erste Notizen zu diesem Thema, die auch Carex erwähnen, finden sich schon in dem Questions and Experiments betitelten Notizbuch, das Darwin in den Jahren von 1839 bis 1844 führte. ${ }^{21}$ Es war also gerade der Umstand, dass sich so viele Naturforscher des Linné'schen Sexualsystems bedienten, der dafür sorgte, dass Geschlechtlichkeit nicht mehr als festgefügte Naturgegebenheit, sondern als variable Anpassungsleistung erschien. ${ }^{22}$

Die Verschränkung von rigider Konvention und penibler Beobachtung, die für die Linné'sche Naturgeschichte kennzeichnend ist und mit Willdenows Handexemplar exemplarisch zu Tage tritt, war also durchaus in der Lage, weitreichende Forschungsfragen $\mathrm{zu}$ generieren. Derselbe Zusammenhang spielt erstaunlicherweise auch für die auf den ersten Blick ausschließlich praktischen Zwecken dienende Nummerierung der Arten im Prodromus eine Rolle. Auf einem Durchschussblatt am Ende des Bandes findet sich eine Rechnung, in der das Zahlenverhältnis von Phanerogamen (Blütenpflanzen) zu Kryptogamen ermittelt wird. ${ }^{23}$ Diese Rechnung scheint die einzige Notiz in 
Willdenows Handexemplar zu sein, die nicht aus seiner Hand stammt. Sie wurde vielmehr von Carl Sigismund Kunth (1788-1850) durchgeführt, ${ }^{24}$ einem kaufmännischen Angestellten am königlichen Seehandlungsinstitut, der von Willdenow an die Botanik herangeführt wurde und 1813, also kurz nach Willdenows Tod, eine eigene Flora Berolinensis herausgab. Sie umfasst zwei Bände, von denen der erste die Blütenpflanzen, der zweite die Kryptogamen behandelt. Im selben Jahr noch zog Kunth nach Paris, wo er bis 1829 als persönlicher Sekretär Alexander von Humboldts dessen botanische Sammlung aus Südamerika in Paris bearbeitete (Wunschmann 1883).

Sicherlich ermittelte Kunth das Verhältnis Phanerogamen zu Kryptogamen in erster Linie, um den ungefähren Umfang der beiden Bände seiner geplanten Flora Berolinensis im Vorfeld einschätzen zu können. Aber auch hier lohnt es sich nachzuhaken. Humboldt und Willdenow hatten sich bereits 1788 kennengelernt und gemeinsam Ideen zu einer Gliederung der Pflanzenwelt in Florengruppen und "Schöpfungscentren" entwickelt, die Willdenow in seinem Grundriss der Kräuterkunde dann als erster veröffentlichte (Jahn 1966). Und in diesen biogeographischen Überlegungen spielten die Proportionen unterschiedlicher taxonomischer Einheiten eine besondere Rolle (Willdenow 1792: 366). Auch in seinem 1799 erschienenen Beitrag zu den Seggen Berlins hatte Willdenow hervorgehoben, dass die Zahl der CarexArten von den Polen zum Äquator abnimmt. Humboldt blieb in seinem Essai sur la géographie des plantes, den er 1805 zusammen mit Aimé Bonpland verfasste, ähnlich qualitativ. In seiner Schrift De distributione geographica plantarum 12 Jahre später erhob er dagegen genaue Verhältniszahlen zum Maßstab einer „botanischen Arithmetik (Arithmetica botanica)" (Humboldt 1817: 18). Nicht nur das Verhältnis von Phanerogamen zu Kryptogamen kommt dabei zur Sprache (ebd.: 28-30), sondern auch der nach Norden anwachsende Anteil von Cyperaceen an den Gräsern insgesamt (ebd.: 202). Der Beitrag des ehemaligen Handelsangestellten Kunth zu diesen Erkenntnissen wird dabei immer wieder hervorgehoben. ${ }^{25}$

Wie es in dem Bewilligungsschreiben des Staatskanzlers Hardenberg an seinen Minister Altenstein hieß, sollte der Ankauf von Willdenows Bibliothek und Herbar die Grundlage bilden, um die „Species Plantarum Willdenovii einer neuen kritischen Durchsicht zu unterwerfen und fortzusetzen", ein Vorhaben, das „der Herr Professor Link ausgesprochen hat [und das] durch den Fleiß und die anerkannten Verdienste [Willdenows] so vielversprechend für die Wissenschaft" sei. ${ }^{26}$ Willdenows Arbeit an den Species plantarum machte ihn zum Mittelpunkt eines weltumspannenden Netzwerks von Korrespondenten und Sammlern (Müller-Wille/Böhme 2010), und auch inhaltlich setzte er mit seinen biogeographischen Überlegungen neue Akzente in der Botanik (Jahn 1966). Aber auch im Kleinen, Alltäglichen und Naheliegenden, so hoffen wir mit unserer Vorstellung von Willdenows Handexemplar gezeigt zu haben, spielte sich der Fortschritt geradezu handgreiflich ab, den 
sich Hardenberg von der Fortsetzung der Species plantarum erhoffte. Das Zusammenspiel von gedruckter Vorlage, handschriftlicher Fortschreibung und Wiederauflage, geregelt durch streng eingehaltene Konventionen, resultierte in einer allmählichen Akkumulation von Fakten, durch die sich mit der Zeit die Umrisse ganz neuer Forschungsgegenstände abzeichneten.

\section{Anmerkungen}

1 Wie erst später festgestellt wurde, war der Name Carex paradoxa bereits zuvor von Johann Friedrich Gmelin (1748-1804) für eine andere Art verwendet worden. Damit war der Name ungültig und die Schwarzschopf-Segge wird heute als Carex appropinquata bezeichnet (siehe The Plant List, 2010, Version 1, http://www.theplantlist.org/ [letzter Zugriff am 10. April 2012]).

2 Wir schließen uns damit der Perspektive an, die auch die übrigen Beiträge in diesem Themenheft Paper Technology. Ein Forschungsinstrument in der Frühen Neuzeit, NTM. Zeitschrift für die Geschichte der Wissenschaften, Technik, Medizin, 21 (2013), annehmen.

3 Willdenow 1787, Staatsbibliothek zu Berlin - Preußischer Kulturbesitz (SBB), Abteilung Historische Drucke, Signatur: $8^{\circ}$ Lx 9406 : R. Im Folgenden zitieren wir diesen Band als „Willd. H.“.

4 SBB, Handschriftenabteilung, Signatur: Ms. Cat. A 188, p. 112 v: "c. annot. manusc. Willden." Der Verweis auf Willdenow fehlt in späteren Katalogen. Als Grundlage für den Handschriftenvergleich diente ein Brief Willdenows vom 1. April 1800, SBB Handschriftenabteilung, Signatur: Sgl. Autographen, Willdenow, Karl Ludwig.

5 „Acta betrifft den Ankauf der Bibliothek des Professor Willdenow de anno 1818“, SBB Handschriftenabteilung, Signatur: Acta III B 14. Die im Zusammenhang mit dem Erwerb der Bibliothek angefertigten Bücherlisten führen unser Exemplar allerdings nicht auf (vgl. ebda., Bl. 12 und 14).

6 Willd. H.: xvi/1. Das Manuskript war vermutlich ursprünglich bloß eingelegt und wurde erst mit der Neubindung über einen eingeklebten Steg mit dem Buchblock verbunden.

7 Ebd.; xvi/1, f. 1v u. 3r.

8 Ebd.: xvi.

9 Ebd.: xvi/1, f. 1 r.

10 Ebd.: xvi/1, f. $5 \mathrm{v}$.

11 Z. B. Willd. H.: 5, „Offic. Cortex Salicis fragilis“ unter „13. Salix fragilis“.

12 Willdenow 1787: passim. Besonders in der Gattung Carex finden sich mehrere durch Abbildungen ergänzte Artbeschreibungen (ebd.: 31-38, tab. I-III).

13 Willd. H.: opp. xvi. Im lateinischen Original: "NB. / In editione altera Genera umbelliferarum et Fungorum emendandae sunt. / Divisio generum in Algis. e.g. Lichen. Verrucar. etc. a certioribus Characteribus petenda est." Das der Seitenzahl vorangestellte „opp.“ gibt hier und im Folgenden an, dass wir aus den handschriftlichen Notizen auf dem der entsprechenden Seite gegenüber befindlichen Durchschussblatt zitieren.

14 Ebd.: opp. 26: „prope Cöpenick et in der Hasenheide copiose. Thiergarten“; opp. 94 „du Roi Harbk. 2 p. 495", ein Verweis auf Johann Philipp DuRois Die Harbkesche wilde Baumzucht (1771-1772).

15 Willd. H.: opp. 122.

16 Ebd.: opp. 166. Der Zettel wurde bei der Neubindung mit eingebunden, weist aber keine früheren Bindungsspuren auf.

17 Ebd.: opp. 78, 122, 281, opp. 415.

18 Carl von Linné stand vor demselben Problem, als er den botanischen Teil der 10. Auflage seines Systema naturae (1758-1759) vorbereitete und neue Arten in die in früheren Auflagen durchnummerierten Artlisten aufnehmen musste. Statt die ursprüngliche 
Nummerierung zu ändern, bezeichnete er neue Arten allerdings durch einen nachgestellten lateinischen Großbuchstaben (Jarvis 2007).

19 Willd. H.: opp. 29. Im lateinischen Original: „Nunquam Caricem tantopere variantem vidi ingenue fateor."

20 Willdenow 1799: 39. Im lateinischen Original: „Observatio: Sexus in hac specie valde inconstans observatur."

21 Charles R. Darwin [1839-1844], Notebook: Questions \& experiments, CUL-DAR206.1, transcribed by Kees Rookmaaker, Darwin Online (http://darwin-online.org.uk/ [letzter Zugriff am 16. April 2012]).

22 Dies steht im Widerspruch zu Londa Schiebingers These, wonach das Sexualsystem zur Verfestigung von Geschlechterkategorien führte (Schiebinger 2004: 20-28).

23 Willd. H.: opp. 422.

24 Der Handschriftenabgleich erfolgte mit einem undatierten, nur als Fragment erhaltenen Schreiben von Kunth an Alexander von Humboldt. In dem Schreiben gibt Kunth Rechenschaft über die Zahl der Arten in einzelnen Pflanzenfamilien. Literaturverweise mit Angabe der Jahreszahl lassen schließen, dass das Schreiben nach 1846 entstanden sein muss (SBB, Handschriftenabteilung, Nachlass A. v. Humboldt, gr. K. 8: Nr. 22).

25 Siehe zu Humboldts „botanischer Arithmetik“ Browne 1983: 58-64.

26 Hardenberg an Altenstein, 15. Juli 1818 (SBB, Handschriftenabteilung, Signatur: Acta III B 14: Bl. 4).

\section{Literatur}

Allgemeines Verzeichnis der Bücher, welche in der Frankfurter und Leipziger Ostermesse des 1787 Jahres neu gedruckt oder aufgelegt worden sind. Leipzig: Weidmann, Oster 1787.

Arenstorff, C. F., 1762. Comparatio Nominum Plantarum Officinalium Cum Nominibus Botanicis Linaei Et Tournefortii. Berlin: Grynäus \& Decker.

Blair, Ann, 2004. Note Taking as an Art of Transmission. Critical Inquiry, 31, 85-107.

Brendecke, Arndt, 2005. „Durchschossene Exemplare“. Über eine Schnittstelle zwischen Handschrift und Druck. Archiv für Geschichte des Buchwesens, 59, 50-64.

Browne, Janet, 1983. The Secular Arc. Studies in the History of Biogeography. New Haven: Yale University Press.

Cooper, Alix, 2007. Inventing the Indigenous. Local Knowledge and Natural History in Early Modern Europe. Cambridge: Cambridge University Press.

Darwin, Charles Robert, 1876. The Effects of Cross and Self Fertilisation in the Vegetable Kingdom. London: John Murray.

Elsholtz, Johann Sigismund, 1663. Flora Marchica, Sive Catalogus Plantarum. Berlin: Runge.

Hiepko, Paul, Hg., 1972. Herbarium Karl Ludwig Willdenow. Alphabetical Index. Zug: Inter Documentation Co.

Humboldt, Alexander von, 1817. De distributione geographica plantarum secundum coeli temperiem et altitudinem montium prolegomena. Paris: In libraria graeco-latina-germanica.

Humboldt, Alexander von/Bonpland, Aimé, 1805. Essai sur la géographie des plantes. Paris: Levrault und Schoell.

Jahn, Ilse, 1966. Carl Ludwig Willdenow und die Biologie seiner Zeit. Wissenschaftliche Zeitschrift der Humboldt Universität Berlin, Mathematisch-naturwissenschaftliche Reihe, 15, 803-812.

Jarvis, Charlie, 2007. A Concise History of the Linnean Society's Linnaean Herbarium, with some Notes on the Dating of the Specimens it Contains. In: Brian Gardiner, Hg., The Linnean Collections. London: The Linnean Society, 5-18.

Linné, Carl von, 1797-1830. Species plantarum. 4. Aufl., Hg. von Karl Ludwig Willdenow und Heinrich Friedrich Link, 6 Bde. Berlin: Nauck.

Müller-Wille, Staffan/Böhme, Katrin, 2010. Biologie. Wissenschaft vom Werden, Wissenschaft im Werden. In: Elmar Tenorth, Hg., Geschichte der Universität Unter den Linden, 1810-2010, Bd. 4, Genese der Disziplinen. Die Konstitution der Universität, Berlin: Akademieverlag, 425446. 
Müller-Wille, Staffan/Charmantier, Isabelle, 2012. Natural History and Information Overload. The Case of Linnaeus. Studies in History and Philosophy of Biological and Biomedical Sciences, 43, 4-15.

Pugliano, Valentina, 2012. Specimen Lists. Artisanal Writing or Natural Historical Paperwork? Isis, 103, im Druck.

Schiebinger, Londa, 2004. Nature's Body. Gender in the Making of Modern Science. 2. Aufl., New Brunswick: Rutgers University Press.

Schlechtendal, Diedrich Franz Leonard, 1814. Karl Ludwig Willdenow. Magazin der Gesellschaft Naturforschender Freunde, 6, v-xvi.

Willdenow, Karl Ludwig, 1787. Florae Berolinensis Prodromus. Berlin: Vieweg.

Willdenow, Karl Ludwig, 1792. Grundriss der Kräuterkunde. Berlin: Haude und Spener.

Willdenow, Karl Ludwig, 1799. Über die in der Gegend von Berlin wildwachsenden RietgrasArten. Sammlung der deutschen Abhandlungen, welche in der Königlichen Akademie der Wissenschaften vorgelesen, 1792-1797, 34-50.

Wunschmann, Ernst, 1883. Kunth, Karl Sigismund. In: Allgemeine Deutsche Biographie. Leipzig: Duncker \& Humblot, 17, 394-397.

\section{Katrin Böhme}

Staatsbibliothek zu Berlin - Preußischer

Kulturbesitz, Abteilung Historische Drucke

Unter den Linden 8

10117 Berlin

Deutschland

E-Mail: katrin.boehme@sbb.spk-berlin.de

Staffan Müller-Wille

University of Exeter, College of Humanities

Amory Building, Rennes DriveExeter

Devon, EX4 4RJ

UK

E-Mail: S.E.W.Mueller-Wille@exeter.ac.uk 